\title{
Résistance à l'insuline dans l'obésité
}

\section{Yannick \\ Le Marchand-Brustel \\ Directeur de recherche à l'Inserm}

Remerciements

Nous remercions A. Grima pour le travail d'illustration de cet article, J. Kubar, N. Rochet et J.-F. Tanti pour leur critique du manuscrit.

Les travaux originaux rapportés dans ce texte ont été financés par l'Inserm, l'Université de Nice, la Fondation pour la Recherche médicale, l'Association des Myopathes de France et le Comité Doyen Jean Lépine (Nice)

\section{RÉFÉRENCES}

1. De Fronzo RA, Tobin JD, Andres R. Glucose clamp technique : a method for quantifying insulin secretion and insulin resistance. Am J Physiol 1979 ; 237 : E214-23.

2. Rizza RA, Mandarino LJ. Gerich JE. Dose-response characteristics for effects of insulin on production and utilization of glucose in man. Am J Physiol 1981 ; 240 : E630-9.

3. Kahn CR. Insulin resistance, insulin insensitivity, and insulin unresponsiveness : a necessary distinction. Melabolism 1978; 27 : 1893-1902.

4. Freychet P. Résistances à l'insuline. Aspects physio-pathologiques et biochimiques. Ann Endocrinol (Paris) 1984 ; 45 : 107-14.

5. Denton RM. Early events in insulin actions. In : Greengard P, Robison GA, eds. Advances in Cyclic Nucleotide and Protein Phosphorylation Research. New York : Raven Press, 1986 ; $20: 293-341$.

6. Czech MP. The nature and regulation of the insulin receptor: structure and function. Annu Rev Physiol 1985; 47 : 357-81.

\section{ADRESSE}

Y. Le Marchand-Brustel : Inserm U.145, faculté de médecine, avenue de Valombrose, 06034 Nice Cedex.

L'obésité est un processus dynamique dans lequel l'hyperinsulinisme, primitif ou secondaire, joue un rôle précoce essentiel. Il aboutit en effet d'abord à un développement rapide du tissu adipeux, puis à l'installation d'une résistance à l'insuline qui est due à la fois à des modifications quantitatives et qualitatives des récepteurs de l'hormone et à une altération des processus qu'elle contrôle.

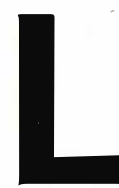

es obésités animales, génétiques ou créées expérimentalement, ainsi que nombre d'obésités humaines, comportent souvent un état de résistance à l'insuline caractérisé in vivo par l'association d'une hyperinsulinémie et d'une glycémie normale ou augmentée. Cette résistance à l'action de l'hormone se retrouve in vitro au niveau de ses principaux tissus cibles tel le tissu adipeux ou le muscle squelettique : des concentrations physiologiques d'insuline produisent un effet biologique diminué. En pratique, le diagnostic de résistance à l'insuline sera donc porté devant une association d'hyperinsulinémie et d'une glycémie normale ou élevée.

\section{Définition et mise en évidence}

La mise en évidence de la résistance à l'insuline peut être réalisée par des tests simples d'hypoglycémie provoquée ou de tolérance au glucose. Le premier consiste à mesurer la réponse hypoglycémique induite par l'injection d'une quantité connue d'insuline. Le deuxième consiste à mesurer la réponse insulinosécrétoire du pancréas à une charge en glucose et la tolérance au glucose de l'organisme. Ces tests présentent deux types de limites; (a) ils ne permettent pas de réaliser des courbes dose-effet complètes de l'insuline, dont l'intérêt sera vu ultérieurement ; (b) l'interprétation du test à l'insuline est difficile du fait de la sécrétion d'hormones contrerégulatrices en réponse à l'hypoglycémie. Plus récemment, pour pallier ces inconvénients, la technique du " clamp " euglycémique hyperinsulinémique a été mise au point, d'abord chez l'homme puis chez l'animal [1]. Ce test permet d'évaluer in vivo la sensibilité globale de l'organisme à l'insuline. $\mathrm{Au}$ cours de cette épreuve, schématiquement représentée dans la figure $1 A$, un niveau constant d'insulinémie est imposé par une perfusion intraveineuse d'insuline ; simultanément la glycémie est maintenue stable par une perfusion de glucose dont le débit est ajusté en fonction des valeurs de glycémie obtenues. La quantité de glucose injecté, nécessaire au maintien de la glycémie pour un niveau d'insulinémie constant, permet de mesurer l'effet de l'insuline sur le métabolisme glucidique de l'organisme (figure 1B). Chez un sujet insuli- 
norésistant, la quantité de glucose perfusé nécessaire au maintien d'une glycémie stable est donc inférieure à celle injectée à un sujet normal. Puisque plusieurs niveaux d'insulinémie sont successivement imposés, il est possible de réaliser des courbes dose-effet complètes et de mesurer ainsi la sensibilité à l'insuline et la réponse maximale à l'hormone. $\mathrm{Si}$ cette technique est couplée à l'utilisation de glucose tritié, il est possible de calculer la production hépatique de glucose (figure $1 C$ ) et l'utilisation périphérique du glucose (figure 1D) [2].

Il est en effet nécessaire de distinguer, dans l'insulinorésistance, la diminution de sensibilité à l'insu-

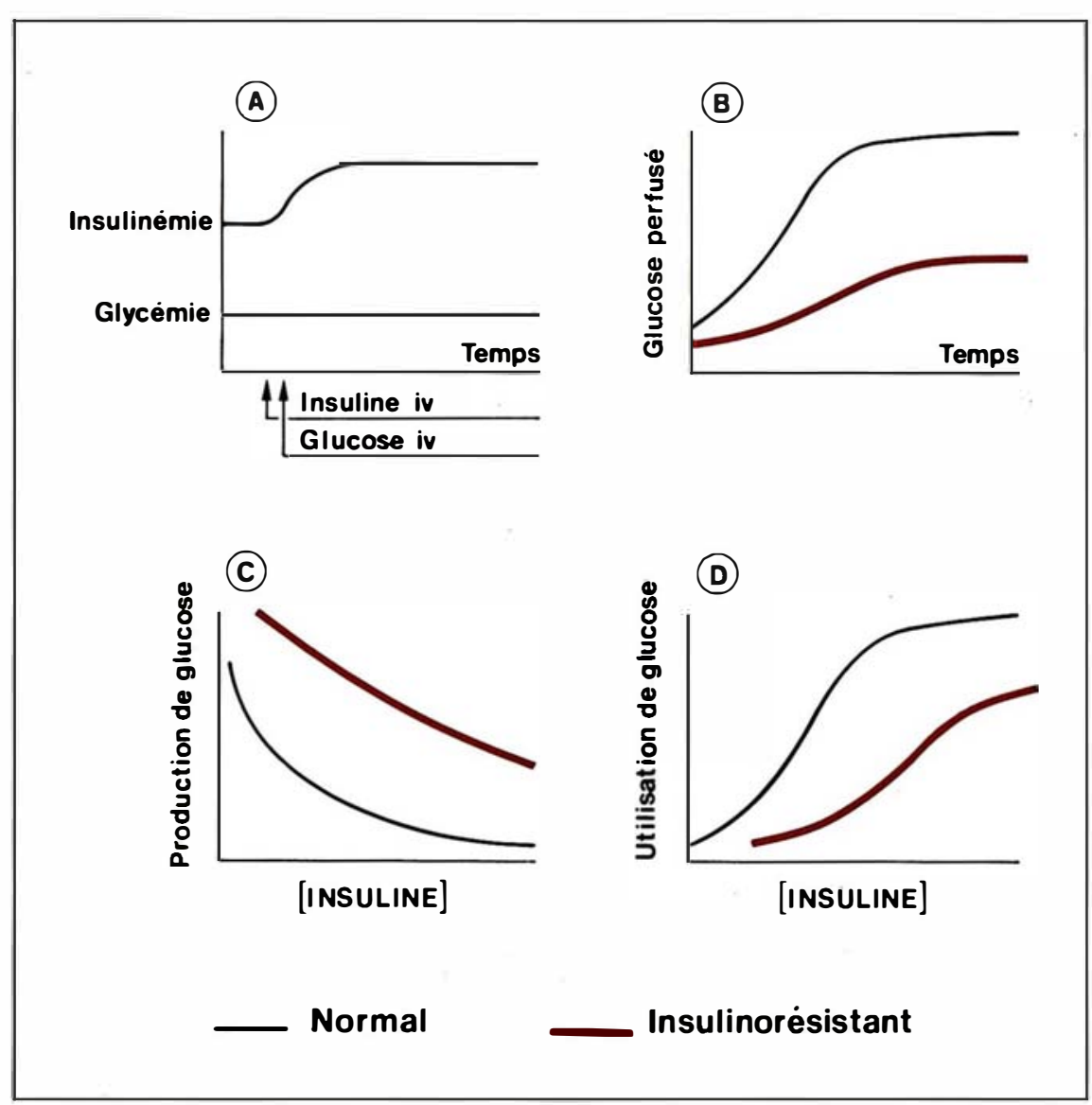

Figure 1. Principe du "clamp" euglycémique hyperinsulinémique. Au cours de cette épreuve, une insulinémie constante est imposée par perfusion intraveineuse (iv) d'insuline. Une minute après l'injection d'insuline, une perfusion de glucose est mise en place, dont le débit est ajusté pour maintenir une glycémie stable en fonction des déterminations du glucose sanguin (A). Le taux de glucose perfusé (B) atteint après quelques minutes est stable et reflète le métabolisme glucidique global de l'organisme. Chez le sujet insulinorésistant, face à une hyperinsulinémie donnée, le métabolisme glucidique est plus faible que chez le sujet normal. Cette épreuve est répétée à différents niveaux d'hyperinsulinémie. En couplant cette technique avec l'utilisation de glucose tritié, il est possible de déterminer la production hépatique de glucose (C) et de calculer l'utilisation périphérique de glucose (D). Chez le sujet insulinorésistant, la production hépatique de glucose n'est pas supprimée par l'insuline et l'utilisation de glucose est plus faible que chez le sujet normal à toutes les concentrations d'insuline (diminution de la réponse maximale). II $y$ a de plus une diminution de la sensibilité à l'insuline, la concentration d'insuline nécessaire à l'obtention de $50 \%$ des effets maximaux (EC50) étant supérieure chez les sujets insulinorésistants à celle obtenue chez les sujets normaux. line de la diminution de réponse maximale à l'hormone [3, 4]. Une diminution de sensibilité est caractérisée par une altération des effets de l'insuline à des concentrations effectives submaximales sans amputation de la réponse maximale, et donc par un déplacement vers les concentrations supérieures de l'ensemble des courbes doseeffet. Ceci résulte le plus souvent d'une simple diminution du nombre des récepteurs. En effet, à l'état normal, il suffit qu'une fraction relativement faible (5-20\%) des récepteurs de l'insuline soit occupée pour que l'action biologique maximale de l'insuline soit exprimée. Les récepteurs dont l'occupation n'est pas nécessaire à l'action maximale de l'insuline sont définis comme des "récepteurs de réserve". Du fait de l'existence de ces récepteurs de réserve, une diminution isolée du nombre des récepteurs (sans changement d'affinité) n'aura pour conséquence qu'un déplacement vers la droite de la courbe doseeffet. Par opposition, une diminution de la réponse maximale est caractérisée par une diminution proportionnellement identique de la réponse à toutes les concentrations (maximales et submaximales) de l'hormone sans modification de la sensibilité. Une telle altération résulte en général d'anomalies indépendantes de la liaison de l'insuline à ses récepteurs. Le plus souvent, comme le montre la figure 1 ( $C$ et $D)$, les deux défauts sont combinés. Cette distinction entre sensibilité et réponse maximale à l'insuline peut permettre de séparer les altérations de liaison de l'insuline à ses récepteurs de celles qui sont postérieures à l'étape de liaison.

Pour étudier l'insulinorésistance associée à l'obésité, on dispose d'un certain nombre de modèles animaux: rats ou souris génétiquement obèses (rat fa/fa de Zucker, souris $o b / o b$ ) ou animaux rendus obèses par destruction chimique (souris rendues obèses par aurothioglucose) ou électrolytique (rats VMH) de l'hypothalamus. Ces modèles d'obésité induite, où le début de la maladie est très 


\section{RÉFÉRENCES}

7. Gammeltoft S, Van Obberghen E. Protein kinase activity of the insulin receptor. Biochem $J 1986 ; 235: 1-11$.

8. Kahn CR, White MF, Grigorescu F, Takayama S, Häring HU, Crettaz M. The insulin receptor protein kinase. In : Czech MP, ed. Molecular Basis in Insulin Action. New York : Plenum Press, 1985 : 67-93.

9. Ebina $\mathrm{Y}$, Ellis L, Jarnagin $\mathrm{K}$ el al. The human insulin receptor cDNA: the structural basis for hormone-activated transmembrane signalling. Cell $1985 ; 40$ : 747-58.

10. Ullrich A, Bell JR, Chen EY, et al. Human insulin receptor and its relationship to the tyrosine kinase family of oncogenes. Nature $1985 ; 313$ : 756-61.

11. Cushman SW, Simpson IA, Smith U Insulin-induced integral membrane protein translocations and their counterregulation by lipolytic and antilipolytic hormones. In : Belfrage P, Donner J, Strälfors P, eds. Mechanism of Insulin Action. Amsterdam : Elsevier Science Publishers, 1986 : 181-210.

12. Tanti JF, Grémeaux T, Brandenburg D Van Obberghen E, Le Marchand-Brustel Y. Brown adipose tissue in lean and obese mice. Insulin-receptor binding and tyrosine kinase activity. Diabeles 1986 ; 35 : 1243-8.

13. Havrankova J, Brownstein MJ, Roth J Concentrations of insulin and of insulin receptors in the brain are independent of periphe ral insulin levels. Studies of obese and streptozotocin-treated rodents. $J$ Clin Inves 1979 ; $64: 636-42$

14. Le Marchand-Brustel $Y$, Jeanrenaud $B$, Freychet $\mathrm{P}$. Insulin binding and effects in isolated soleus muscle of lean and obese mice. $A m$ $J$ Physiol 1978 ; 234 : E348-58.

15. Wardzala LJ, Jeanrenaud B. Potentia mechanism of insulin action on glucose trans port in the isolated rat diaphragm. Apparent translocation of intracellular glucose transport units to the plasma membrane. I Biol Chem $1981 ; 256: 7090-3$

16. Le Marchand-Brustel $Y$, Freychet $P$ Regulation of glycogen synthase activity in the isolated soleus muscle. Effect of insulin, epinephrine, glucose and anti-insulin receptor antibodies. Biochim Biophys Acta 1981; 677 : 13-22.

17. Oron Y, Larner J. Insulin action in intact mouse diaphragm. Activation of glycogen synthase through stimulation of glucose trans port and phosphorylation. Mol Cell Biochem 1980 ; 32 : 153-60.

18. Le Marchand-Brustel Y, Freychet P. Alteration of glycogen synthase activation by insulin in soleus muscles of obese mice. FEBS Lett. $1982 ; 120$ : 205-8

19. Crettaz M, Prentki M, Zaninetti D, Jeanrenaud $B$. Insulin resistance in soleus muscle from obese Zucker rats. Involvement of several defective sites. Biochem $J 1980 ; 186$ : précisément connu, permettent l'étude de l'aspect évolutif du syndrome. Il existe également des modèles animaux où l'obésité est induite par des régimes alimentaires riches en graisses, en glucides ou rendus attractifs par leur diversité (régime " cafeteria").

\section{Mécanisme d'action de I'insuline}

La résistance à l'insuline peut résulter d'altérations intervenant aux différentes étapes de l'action de l'insuline. Nous commencerons donc par résumer brièvement l'état actuel des connaissances du mécanisme d'action de l'insuline, qui reste d'ailleurs en partie non élucidé [5-8] (figure 2). L'insuline exerce ses effets biologiques après liaison à des récepteurs membranaires spécifiques. Cette interaction de l'insuline avec son récepteur conduit à une grande variété de réponses biologiques sur des transports membranaires, des enzymes intracellullaires, la synthèse protéique ou la croissance cellulaire, comme le représente très schématiquement la figure 2. Le récepteur est une glycoprotéine tétramérique composée de deux sous-unités $\alpha$ de masse moléculaire $130 \mathrm{kD}$ qui portent le ou les sites de liaison de l'insuline et sont totalement extracellulaires, et de deux sousunités $\beta$ de masse moléculaire $95 \mathrm{kD}$, qui sont transmembranaires. La séquence en acides aminés du récepteur de l'insuline a été récemment déduite de la séquence en nucléotides de l'ADN complémentaire de son précurseur $[9,10]$. Une avance importante dans la compréhension du mécanisme d'action de l'insuline a été réalisée avec la découverte de la présence d'une activité tyrosine kinase portée par cette sousunité $\beta$ du récepteur [6-8]. L'insuline stimule l'autophosphorylation de cette sous-unité dans des systèmes acellulaires de récepteurs ou dans des cellules intactes. L'activité kinase du récepteur s'exerce également sur des protéines exogènes. Bien que divers substrats endogènes poten- tiels de la kinase du récepteur insulinique aient été récemment décrits, le mécanisme exact de la transmission du message hormonal n'est pas encore totalement élucidé, en particulier le rôle de cette activité enzymatique portée par la sous-unité $\beta$ du récepteur [5-8]. En effet, le récepteur est une tyrosine kinase, tandis que la plupart des phosphorylations des protéines cellulaires interviennent sur des résidus sérine ou thréonine. D'autre part, certaines enzymes régulées par l'insuline sont activées par phosphorylation, telle l'acétyl CoA carboxylase, d'autres par déphosphorylation telles que la pyruvate déshydrogénase ou la glycogène synthétase [5]. Enfin, l'insuline stimule la translocation de certaines protéines comme les transporteurs du glucose ou le récepteur de l'IGF II (facteur de croissance insulinomimétique de type II), aucune phosphorylation n'ayant été mise en évidence au cours de cette translocation [11]. Enfin, comme le montre la figure 2, les localisations intracellulaires des cibles de l'insuline sont variées : membrane plasmique, ribosomes, mitochondries, cytosol, noyau. Ceci implique qu'il n'y a pas de schéma unique actuellement connu pour expliquer les effets de l'insuline.

\section{Altérations observées}

(a) Diminution du nombre des récepteurs de l'insuline. La première altération rapportée dans les obésités humaines ou animales avec insulinorésistance a été une diminution de la liaison de l'insuline à ses principaux tissus cibles, due essentiellement à une diminution du nombre des récepteurs sans modification de leur affinité $[3,4]$. Cette diminution de liaison de l'hormone est généralement attribuée au phénomène de down-regulation, ou régulation négative des récepteurs induite par l'insuline elle-même, phénomène amplement documenté au niveau de cellules en culture. Le facteur essentiel contrôlant le nombre des récepteurs insuliniques dans les 


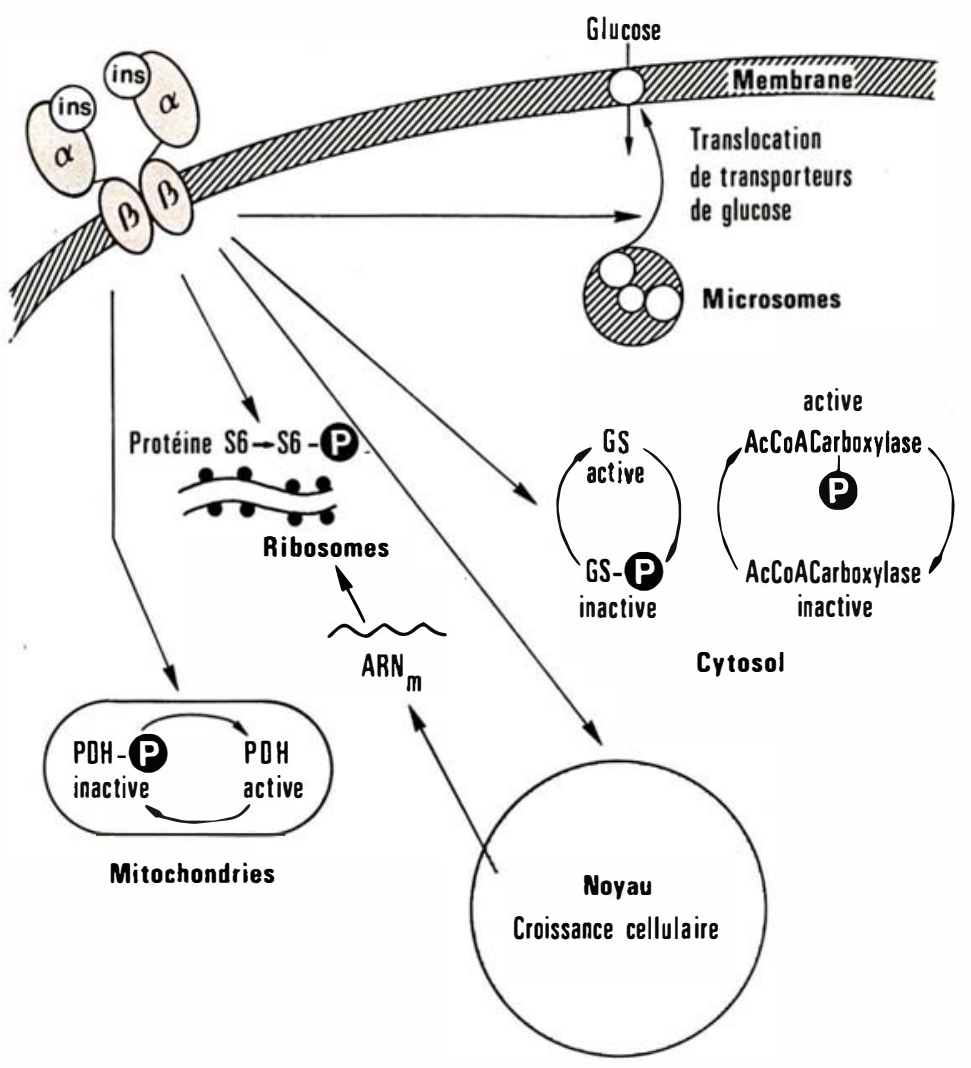

Figure 2. Schéma du mécanisme d'action de I'insuline. L'insuline (ins) se fixe sur la sous-unité $\alpha$ et stimule l'activité tyrosine kinase portée par la sousunité $\beta$ du récepteur de l'insuline. L'insuline induit la translocation des transporteurs du glucose depuis le pool intracellulaire vers la membrane plasmique : elle active des enzymes cytosoliques telles la glycogène synthétase (GS) par déphosphorylation et l'acétyl CoA Carboxylase (AcCoACarboxylase) par phosphorylation; elle augmente le niveau de phosphorylation de la protéine ribosomale S6 ; elle favorise la croissance cellulaire et elle active la pyruvate déshydrogénase (PDH) intramitochondriale, la forme active étant également déphosphorylée. $P=$ résidu phosphate.

tissus cibles semble donc être le niveau d'insuline circulante : la diminution du nombre des récepteurs est une réponse - et probablement une protection - de l'organisme contre l'hyperinsulinémie marquée existant chez les animaux obèses. Il faut toutefois noter que le nombre des récepteurs de l'insuline ne diminue pas dans les mêmes proportions dans tous les tissus. Ainsi, la diminution du nombre des récepteurs insuliniques est modérée (30$\mathrm{m} / \mathrm{s} n^{\circ} 7$ vol. 3 , septembre 87 des récepteurs de l'insuline est un déplacement vers la droite de l'ensemble des courbes doseréponse des effets biologiques et donc une diminution de la sensibilité du tissu ou de l'organisme à l'insuline [3,4]. Ce phénomène est illustré dans la figure 3 : la concentration d'insuline nécessaire pour obtenir le demi-effet maximal sur le transport du glucose ou des acides aminés est de l'ordre de $0,5 \mathrm{nM}$ dans les muscles de souris normales et de $1-1,5 \mathrm{nM}$ dans les muscles de souris obèses. Cette différence, bien que modérée, est probablement importante puisque ces concentrations d'insuline sont physiologiques.

(b) Altérations postérieures au récepteur de l'insuline. La modification de la réponse biologique à des concentrations maximales d'insuline est le témoin d'altérations que nous allons passer en revue et qui sont indépendantes de la liaison. Nous nous attacherons plus particulièrement aux anomalies décrites dans le muscle squelettique qui est l'une des cibles essentielles de l'insuline et l'un des principaux tissus utilisateurs de glucose. La première étape du métabolisme du glucose est son transport à travers la membrane cellulaire. Dans le muscle squelettique des animaux obèses insulinorésistants, le transport du glucose est altéré : ainsi, mesuré en absence ou en présence de concentrations maximales d'insuline, il est plus faible dans le muscle de la souris obèse que dans celui de la souris normale (figure 3A) [14]. Le glucose est transporté dans la cellule par des transporteurs spécifiques localisés à sa surface. On retrouve également un grand nombre de transporteurs de glucose à l'intérieur de la cellule au niveau de ce qu'il est convenu d'appeler la fraction microsomale. Dans les cellules adipeuses et musculaires, l'insuline induit la translocation des transporteurs de glucose depuis les compartiments intracellulaires vers la membrane plasmique: sous l'action de l'insuline, le nombre des transporteurs localisés dans les membranes plasmiques augmente 
alors que leur nombre diminue dans le compartiment intracellulaire $[11,15]$. Une diminution du nombre total des transporteurs de glucose a été démontrée dans les cellules adipeuses du rat âgé obèse ou rendu insulinorésistant par un régime hyperlipidique, ainsi que dans les cellules cardiaques de rats génétiquement obèses. Il est vraisemblable, par analogie, qu'une telle diminution est responsable de l'altération du transport de glucose dans le muscle squelettique des animaux obèses insulinorésistants.

Après captation par la cellule musculaire, le glucose est utilisé par la voie glycolytique ou stocké sous forme de glycogène. La régulation par l'insuline de la synthèse de glycogène s'effectue essentiellement au niveau de la glycogène synthétase dont l'activité dépend de l'état de phosphorylation, la forme active étant la forme déphosphorylée. L'insuline active l'enzyme, indépendamment de son effet sur le transport de glucose $[16,17]$. Dans le muscle des souris obèses, alors que la quantité totale d'enzyme et son activité basale sont normales, l'activation de la glycogène synthétase par l'insuline est défectueuse, comme l'illustre la figure $3 B$ [18].

L'utilisation du glucose par la voie glycolytique est diminuée en absence et en présence d'insuline dans les muscles d'animaux obèses. Si cela reflète en partie les altérations du transport du glucose, d'autres facteurs semblent également intervenir. En effet, certains métabolites intracellulaires inhibent l'utilisation du glucose. L'utilisation de substrats lipidiques par le muscle est accrue chez les animaux obèses aux dépens des substrats glucidiques. Les concentrations intracellulaires de citrate et de glucose-6phosphate sont augmentées, entraînant une diminution de la glycolyse par suite de l'inhibition de la phosphofructokinase, enzyme limitante dans la voie glycolytique [9]. Il a été de plus montré que la proportion de forme active de la pyruvate déshydrogénase, autre enzyme
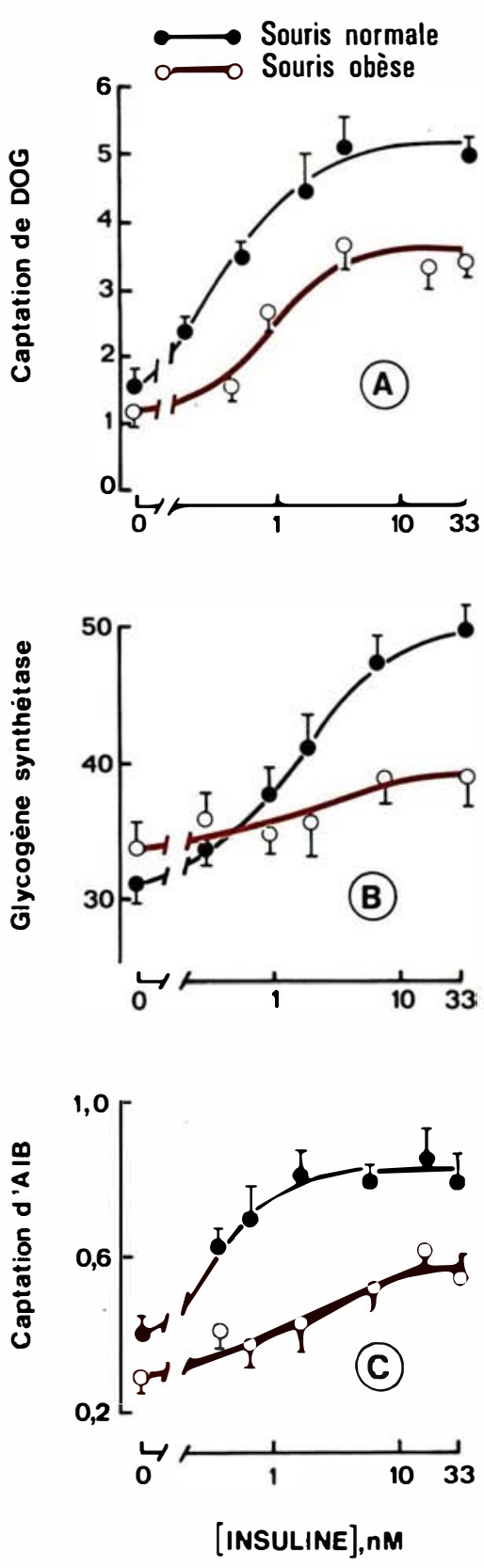

Figure 3. Effet de I'insuline sur la captation de glucose, I'activation de la glycogène synthétase et l'influx d'acides aminés dans lo muscle soleus isolé. Les muscles soleus de souris normales ou rendues obèses par aurothioglucose sont incubés dans un tampon Krebs Ringer Bicarbonate, contenant 1 \% d'albumine bovine, $2 \mathrm{mM}$ de pyruvate et des concentrations variables d'insuline. Panneau A : la captation de désoxyglucose (DOG), analogue non métabolisable du glucose, est mesurée sur une période de 15 minutes et les résultats sont exprimés en nmoles captées par $\mathrm{mg}$ de protéines musculaires pendant ces 15 min. Panneau $B$ : à la fin de l'incubation, les muscles sont homogénéisés pour la détermination de la glycogène synthétase (formes active et totale) ; le pourcentage de la forme active est représenté dans les graphiques. Panneau $C$ : les muscles sont incubés 30 min avec l'acide $\alpha$-aminoisobutyrique (AIB), analogue non métabolisable de I'alanine. La captation musculaire d'AIB est exprimée en nmoles par $\mathrm{mg}$ de protéines musculaires en 30 min ladapté des références $[14,18,21])$. 
limitante de l'utilisation de glucose, est diminuée dans le cœur des souris obèses. Toutes ces anomalies dans le transport du glucose, la synthèse de glycogène et son utilisation par la voie glycolytique et oxydative, vont contribuer à diminuer l'utilisation musculaire du glucose tant à l'état basal qu'en réponse à l'insuline, et donc à augmenter la glycémie.

Le rôle de l'insuline dans le métabolisme protéique musculaire est connu depuis longtemps : elle stimule la synthèse et diminue la dégradation des protéines chez l'animal normal, à la fois in vitro et in vivo [20]. Chez le sujet obèse, en revanche, on observe, en dépit de l'hyperinsulinisme, une diminution relative ou absolue de sa masse musculaire. Le mécanisme qui en est responsable n'est pas tout à fait clair. D'une part, le transport membranaire des acides aminés présente, dans le muscle soleus des animaux obèses, des anomalies très semblables à celles décrites pour le transport du glucose : diminution du transport basal, mesuré en absence d'insuline, dû à une diminution du nombre des transporteurs des acides aminés, diminution de la sensibilité à l'insuline et diminution de la réponse maximale à l'hormone (figure 3C) [21]. D'autre part, le métabolisme protéique global n'est pas altéré à l'état basal ou après stimulation par l'insuline dans les muscles isolés de souris rendues obèses par aurothioglucose [22]. Il ressort de ces études que les anomalies observées in vivo chez les animaux obèses ne sont apparemment pas liées à un défaut proprement dit des processus de synthèse et de dégradation protéique mais que d'autres facteurs sont vraisemblablement impliqués dans la diminution de la masse protéique chez les animaux obèses, notamment la réduction de leur activité physique. L'ensemble des anomalies retrouvées dans le muscle squelettique des animaux obèses insulinorésistants est schématisé dans la figure 4.

(c) Altération des propriétés tyrosine kinase du récepteur de $\mathrm{m} / \mathrm{s} n^{\circ} 7$ vol. 3, septembre 87 l'insuline. La multiplicité des défauts observés dans les obésités avec insulinorésistance suggère qu'une étape très précoce dans le mécanisme d'action de l'insuline est altérée. La découverte de l'activité tyrosine kinase portée par le récepteur de l'insuline a conduit à penser qu'une altération de la phosphorylation du récepteur de l'insuline et de ses propriétés kinasiques pourrait expliquer l'insulinorésistance du muscle squelettique des animaux obèses. Nous avons donc comparé les propriétés de préparations partiellement purifiées de récepteurs insuliniques, obtenues à partir de muscles d'animaux témoins ou d'animaux obèses. Nous avons observé qu'il existe, dans les préparations de récepteurs insuliniques obtenues à partir d'animaux obèses, un défaut d'activation de la tyrosine kinase du récepteur par l'insuline [23]. En effet, pour une même quantité de récepteurs appréciée par la capacité de liaison, l'autophosphorylation et les propriétés kinasiques vis-à-vis d'un substrat synthétique des récepteurs préparés à partir d'animaux obèses sont plus faibles que celles de récepteurs préparés à partir d'animaux normaux. Une altération similaire des propriétés kinasiques des récepteurs insuliniques a ensuite été rapportée dans des adipocytes d'obèses présentant un diabète non insulinodépendant [24]. Le mécanisme de cette altération des propriétés kinasiques des récepteurs insuliniques de sujets insulinorésistants n'est pas encore élucidé. Il est toutefois important de noter que cette altération n'existe que chez les animaux obèses et insulinorésistants [23]. Chez les animaux dont l'obésité est récente et qui ne présentent encore pas de résistance à l'insuline, la diminution de l'activité tyrosine kinase des récepteurs insuliniques ne reflète que l'ampu-

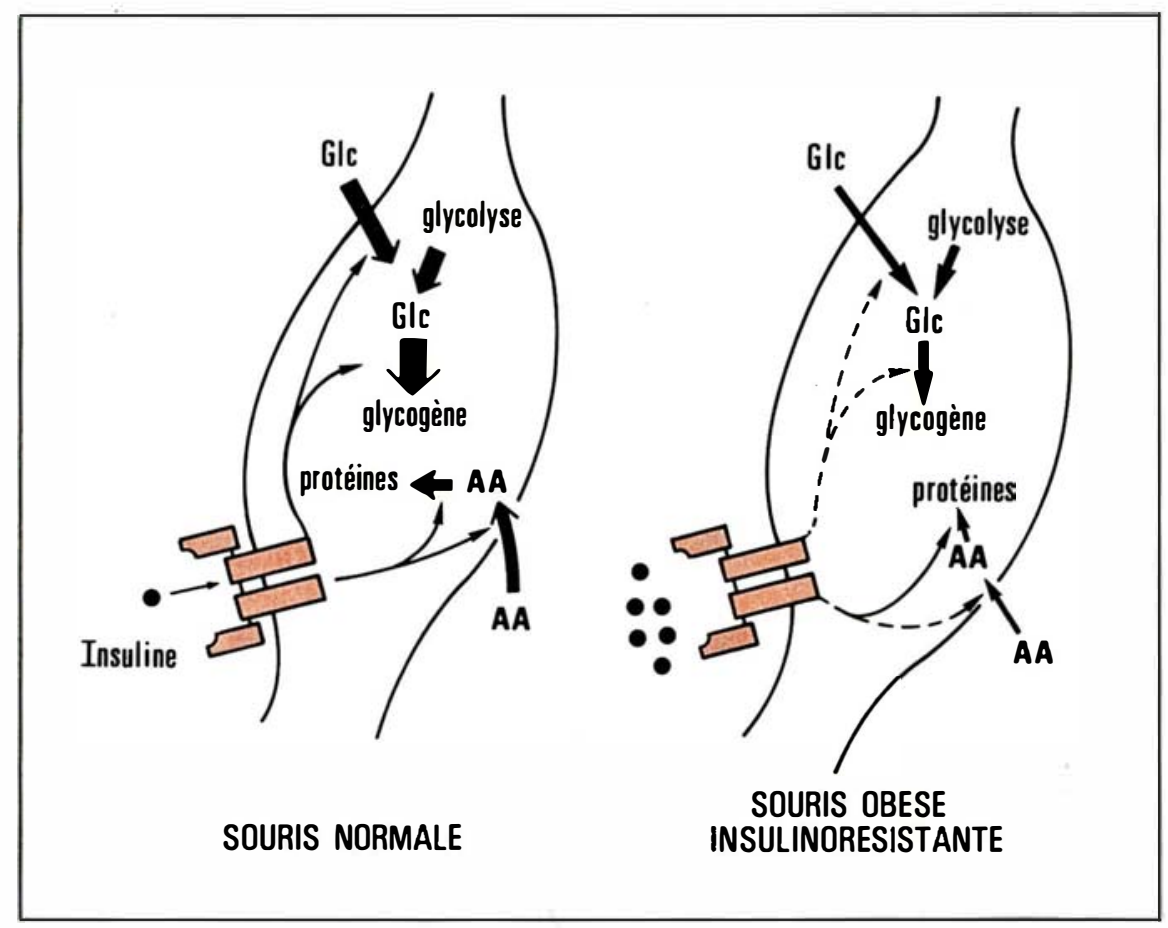

Figure 4. Schéma des anomalies observées au niveau du muscle squelettique de la souris obèse insulinorésistante. De multiples anomalies concourent à l'insulinorésistance du muscle squelettique de la souris obèse : le nombre des récepteurs de l'insuline est diminué d'environ $30 \%$ et les propriétés kinasiques des récepteurs restants sont altérées; le transport basal du glucose et des acides aminés est diminué ; l'activation de la glycogène synthétase par l'insuline est défectueuse. Glc = glucose ; $A A=$ acides aminés. 


\section{RÉFÉRENCES}

20. Jefferson LS. Role of insulin in the regulation of protein synthesis. Diabeles 1980 ; 29 : 487-96.

21. Le Marchand-Brustel $Y$, Moutard N, Freychet P. Aminoisobutyric acid transport in soleus muscles of lean and gold thioglucoseobese mice. Am J Physiol 1982 ; 243 : E74-9.

22. Monier S, Le Cam A, Le MarchandBrustel Y. Insulin and Insulin-like Growth Factor I. Effects on protein synthesis in isolated muscles from lean and goldthioglucose-obese mice. Diabetes 1983 ; 32 : 392-7.

23. Le Marchand-Brustel $Y$, Grémeaux $T$, Ballotti R, Van Obberghen E. Insulin receptor tyrosine kinase is defective in skeletal muscle of insulin-resistant obese mice. Nature 1985 ; $315: 676-9$.

24. Freindenberg GR, Henry RR, Klein $\mathrm{HH}$, Reichart DR, Olefsky JM. Decreased kinase activity of insulin receptors from adipocytes of non-insulin-dependent diabetic subjects. $J$ Clin Invest $1987 ; 79$ : 240.50.

25. Guerre-Millo M, Lavau M, Horne JS, Wardzala LJ. Proposed mechanism for increased insulin-mediated glucose transport in adipose cells from young, obese Zucker rats. Large intracellular pool of glucose transporters. J Biol Chem 1985; 261 : 2197-201.

26. Pénicaud L, Rohner-Jeanrenaud F, Jeanrenaud $B$. In vivo metabolic changes as studied longitudinally after vendromedial hypothalamic lesions. Am J Physiol 1986 ; 250 : E662-8.

27. Debant A, Guerre-Millo $M$, Le Marchand-Brustel Y, Freychet P, Lavau M, Van Obberghen $E$. Insulin receptor kinase is hyperresponsive in adipocytes of young obese Zucker rats. $A m J$ Physiol 1987 ; 252 : E273-78.

28. Le Marchand $Y$, Freychet P, Jeanrenaud $B$. Longitudinal study on the establishment of insulin resistance in hypothalamic obese mice. Endocrinology 1978 ; 102 : 74-85.

29. Jeanrenaud B. An hypothesis on the aetiology of obesity : dysfunction of the central nervous system as a primary cause. Diabetologia $1985 ; 28: 502-13$

30. Ricquier D, Mory G. Factors affecting brown adipose tissue activity in animals and man. Clin Endocrinol Metab 1984; 13 : 501-20.

31. Himms-Hagen J. Thermogenesis in brown adipose tissue as an energy buffer. Implications for obesity. $N$ Engl J Med 1984, 311 : 1549-58.

32. Ricquier D. Thermogenèse et obésité mécanismes moléculaires. médecine/sciences 1985 ; 1 : 147-53.

33. Ferré $\mathrm{P}$, Burnol AF, Leturque $\mathrm{A}$, et al Glucose utilization in vivo and insulinsensitivity of rat brown adipose tissue in various physiological and pathological conditions. Biochem J 1986 ; 233 : 249-52.

34. Arch JRS, Ainsworth AT, Cawthorne MA, et al. Atypical $\beta$-adrenoreceptor on brown adipocytes as target for anti-obesity drugs.
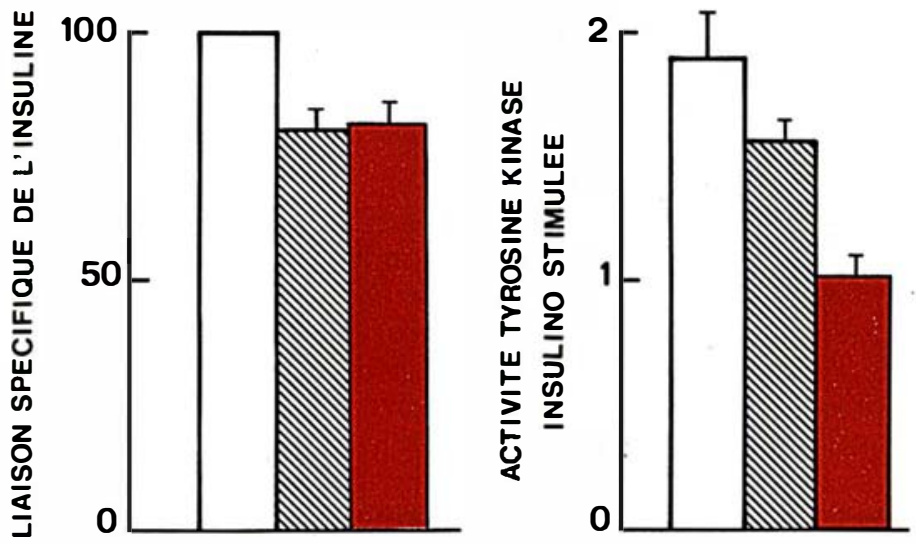

\section{Souris normales}

Souris obèses non insulinoresistantes

Souris obèses insulinorèsistantes

Figure 5. Altération des propriétés tyrosine kinase du récepteur de l'insuline dans le muscle des souris obèses insulinorésistantes. Les récepteurs insuliniques sont partiellement purifiés à partir du muscle squelettique de souris normales ou obèses. Leur capacité de liaison de l'insuline radioiodée est mesurée et leurs propriétés kinasiques sont déterminées sur un substrat synthétique, le copolymère (glutamate-tyrosine, $4 ; 1$ ). La liaison spécifique de l'insuline est exprimée en pourcentage de la liaison obtenue chez les souris normales et l'activité tyrosine kinase stimulée par l'insuline en nmoles de phosphate incorporées sur le copolymère en 30 min. Deux groupes de souris obèses ont été étudiés : des souris non insulinorésistantes (dont l'obésité est d'installation récente et donc en phase dynamique) et des souris insulinorésistantes (à la phase statique de l'obésité). Chez les souris obèses non insulinorésistantes, la diminution de l'activité tyrosine kinase des récepteurs de l'insuline stimulée par l'hormone reflète simplement la diminution du nombre de ces récepteurs. En revanche, chez les animaux insulinorésistants, la diminution d'activité kinase est supérieure à la diminution de liaison, indiquant que les propriétés kinasiques des récepteurs insuliniques sont altérées (adapté de [23]).

tation du nombre des récepteurs. En revanche, chez les animaux obèses plus âgés et insulinorésistants, la diminution de l'activité tyrosine kinase des récepteurs insuliniques est supérieure à celle du nombre des récepteurs (figure 5). De la même façon, les propriétés kinasiques des récepteurs préparés à partir d'adipocytes de sujets obèses, mais non diabétiques, ne sont pas modifiées par rapport à celles de sujets normaux [24].

Ces résultats suggèrent fortement que l'activité kinasique portée par le récepteur de l'insuline joue un rôle important dans le mécanisme d'action de l'hormone, et que son altération participe à l'insulinorésistance liée à l'obésité.

\section{Caractère évolutif du syndrome obèse}

Des études récentes, utilisant des modèles animaux, ont clairement établi que l'obésité est un syndrome évolutif : une obésité d'installation récente est totalement différente d'une obésité ancienne. Ainsi, chez des rats rendus obèses par lésion de l'hypothalamus et étudiés précocement (une semaine) après la lésion, ou chez de jeunes rats Zucker de 30 jours, le foie et le tissu adipeux présentent une augmentation de la 
sensibilité et de la réponse maximale à l'insuline par rapport aux animaux normaux [25, 26]. Plusieurs phénomènes ont été observés au niveau du tissu adipeux : (a) le nombre des transporteurs du glucose est plus élevé dans les adipocytes de jeunes rats Zucker obèses que chez les rats normaux des mêmes portées [25] ; (b) l'activité kinase des récepteurs de l'insuline des adipocytes d'animaux obèses est supérieure à celle des récep- teurs des animaux normaux du même âge [27].

Il semble donc, dans ces modèles et probablement aussi chez l'homme, que la séquence suivante d'événements intervienne, aboutissant à l'obésité puis à l'insulinorésistance : (a) hypersécrétion d'insuline permanente ou transitoire dont la cause peut être variable (désordre hypothalamique, hyperphagie...) ; (b) dans un premier temps, les tissus cibles de

Tableau I

CARACTĖRE ÉVOLUTIF DU SYNDROME OBĖSE HYPERGLYCÉMIQUE

\begin{tabular}{|c|c|c|c|c|}
\hline & $\begin{array}{l}\text { Phase } \\
\text { précoce }\end{array}$ & Réf. & $\begin{array}{c}\text { Phase } \\
\text { tardive }\end{array}$ & Réf. \\
\hline $\begin{array}{l}\text { Insulinémie } \\
\text { Glycémie }\end{array}$ & $\mathrm{N}$ ou & $\begin{array}{l}{[28,29]} \\
{[28,29]}\end{array}$ & $N$ ou $A$ & $\begin{array}{l}{[28,29]} \\
{[28,29]}\end{array}$ \\
\hline \multicolumn{5}{|l|}{ Foie } \\
\hline $\begin{array}{l}\text { Métabolisme } \\
\text { - basal } \\
\text { - insulinostimulé }\end{array}$ & & $\begin{array}{l}{[26,28]} \\
{[26]}\end{array}$ & & $\begin{array}{c}{[26,28]} \\
{[26]}\end{array}$ \\
\hline $\begin{array}{l}\text { Récepteurs de l'insuline } \\
\text { - Nombre } \\
\text { - Activité kinase }\end{array}$ & & [28] & & [28] \\
\hline Muscle squelettique & & & & \\
\hline $\begin{array}{l}\text { Métabolisme } \\
\quad \text { - basal } \\
\text { - insulinostimulé }\end{array}$ & $N$ & $\begin{array}{l}{[26,28]} \\
{[14,28]}\end{array}$ & & $\begin{array}{l}{[26,28]} \\
{[19,28]}\end{array}$ \\
\hline $\begin{array}{l}\text { Récepteurs de l'insuline } \\
\text { - Nombre } \\
\text { - Activité kinase }\end{array}$ & & $\begin{array}{l}{[23,27]} \\
{[23,27]}\end{array}$ & & $\begin{array}{c}{[14,19,23]} \\
{[23]}\end{array}$ \\
\hline Tissu adipeux blanc & & & & \\
\hline $\begin{array}{l}\text { Métabolisme } \\
\quad \text { - basal } \\
\text { - insulinostimulé }\end{array}$ & & $\begin{array}{l}{[25,28]} \\
{[25]}\end{array}$ & & $\begin{array}{l}{[28]} \\
{[28]}\end{array}$ \\
\hline $\begin{array}{l}\text { Récepteurs de l'insuline } \\
\text { - Nombre } \\
\text { - Activité kinase }\end{array}$ & & $\begin{array}{l}{[25,27]} \\
{[27]}\end{array}$ & & $\begin{array}{l}{[24]} \\
{[24]}\end{array}$ \\
\hline Tissu adipeux brun & & & & \\
\hline $\begin{array}{l}\text { Métabolisme } \\
\qquad \begin{array}{l}\text { - basal } \\
- \text { insulinostimulé }\end{array}\end{array}$ & $?$ & & & [33] \\
\hline $\begin{array}{l}\text { Récepteurs de l'insuline } \\
\text { - Nombre } \\
\text { - Activité kinase }\end{array}$ & 1 & $\begin{array}{l}{[12]} \\
{[12]}\end{array}$ & & $\begin{array}{l}{[12]} \\
{[12]}\end{array}$ \\
\hline
\end{tabular}

augmentation modérée ; $1 /$ : augmentation importante ;

diminution modérée; $\forall$ : diminution importante; $N$ : normal. l'insuline, foie et tissu adipeux, répondent normalement ou même de façon exagérée à cette hyperinsulinisme, la sensibilité des tissus étant normale : le tissu adipeux se développe alors très rapidement. Cette phase correspond à la phase dynamique de l'obésité ; (c) apparition progressive d'une diminution de la sensibilité et de la réponse maximale des tissus à l'insuline. L'obésité se stabilise, cette phase statique s'accompagnant d'insulinorésistance. Un cercle vicieux s'établit alors : si la fonction de la cellule B pancréatique n'est pas altérée, la conséquence de la résistance à l'insuline sera une augmentation de la sécrétion pancréatique d'insuline pour compenser la diminution de réponse des tissus. Cette augmentation de sécrétion d'insuline aggrave l'insulinorésistance : plus l'insulinémie s'élève, plus le nombre de récepteurs insuliniques diminue et moins la réponse des tissus à l'insuline est importante. Dans un premier temps, la glycémie reste normale au prix de cette hyperinsulinémie puis, chez certains sujets, un diabète franc apparaît $[28,29]$. L'évolution de tous les tissus n'est pas rigoureusement parallèle : chez de jeunes animaux obèses, le tissu adipeux blanc répond de façon exagérée à l'insuline, cette réponse expliquant le développement explosif de la masse adipeuse, alors que dans le même temps, le muscle squelettique présente déjà une certaine résistance à l'action de l'insuline $[27,28]$. Le Tableau I, présenté ci-contre, résume les principaux résultats établis actuellement dans les différents tissus cibles de l'organisme.

\section{Causes de I'insulinorésistance}

Il est maintenant bien établi que l'hyperinsulinémie est un facteur déterminant de l'obésité et de l'insulinorésistance. La quasitotalité des défauts observés disparaît lorsque l'on abaisse l'insuline circulante par des régimes hypocaloriques, par le jeûne ou en détruisant les cellules B du pan- 
créas par la streptozotocine : le nombre des récepteurs de l'insuline remonte, la réponse des tissus à l'hormone redevient normale, la résistance à l'insuline diminue, ceci indiquant que l'hyperinsulinémie est une cause majeure de la résistance à l'insuline [29]. L'origine de l'hyperinsulinémie est par contre plus obscure. L'hyperphagie aggrave le syndrome de façon indéniable mais n'est pas la seule explication de l'obésité avec insulinorésistance ; en effet l'obésité et l'insulinorésistance peuvent être obtenues sans hyperphagie chez des rats lésés au niveau de l'hypothalamus. De nombreuses données renforcent l'hypothèse de l'existence d'un axe système nerveux central-pancréas, dont le dérèglement aboutirait à l'hyperinsulinémie [29]. Cet aspect du problème fait l'objet d'un article de synthèse dans ce même numéro (artice de B. Jeanrenaud).

Un défaut de la thermogenèse semble également participer au développement de l'obésité [30-32]. Le tissu adipeux brun, tissu spécialisé dans la dissipation de chaleur, présente en effet des anomalies dans la plupart des modèles d'animaux obèses : une diminution de la thermogenèse induite par le froid ou par la prise alimentaire peut expliquer une prise de poids sans hyperphagie (voir, dans ce même numéro, l'article de synthèse de J. Seydoux). Le tissu adipeux brun est un tissu utilisateur de glucose, extrêmement sensible à l'insuline chez l'animal normal et qui pourrait ainsi contribuer de façon significative à l'homéostasie glucidique de l'organisme [33]. Ce tissu contient de nombreux récepteurs de l'insuline chez l'animal normal. $\mathrm{Au}$ cours du développement de l'obésité, le nombre de ces récepteurs est diminué très précocement et dans des proportions très importantes [12]. En effet, bien que le tissu adipeux brun soit hypertrophié chez les animaux obèses, le nombre total des récepteurs de l'insuline exprimé par animal est diminué de plus de $60 \%$ par rapport aux animaux normaux. Cette diminution des récepteurs de l'insuline dans le tissu brun des animaux obèses pourrait expliquer en partie l'obésité et l'insulinorésistance de ces animaux. En effet, une série d'agents $\beta$-agonistes a été récemment développée, qui agissent plus spécifiquement au niveau du tissu adipeux brun en stimulant la thermogenèse [34]. Le traitement par l'une de ces substances de souris rendues obèses par aurothioglucose, a induit une normalisation de la glycémie et une perte de poids associée à la normalisation du nombre des récepteurs de l'insuline dans le tissu adipeux brun (N. Rochet, J.F. Tanti, T. Grémeaux, E. Van Obberghen et Y. Le MarchandBrustel, soumis pour publication). Ces expériences suggèrent que le tissu adipeux brun joue un rôle important dans le maintien d'une glycémie normale chez les rongeurs et elles ouvrent des perspectives intéressantes pour le traitement de l'obésité. Il reste toutefois à prouver que le tissu adipeux brun joue également un rôle prépondérant dans la thermogenèse chez l'homme.

En conclusion, l'obésité et l'insulinorésistance forment un syndrome évolutif et complexe.

Des anomalies, tant au niveau du récepteur de l'insuline (diminution de leur nombre et altération de leurs propriétés kinasiques) que d'étapes indépendantes du récepteur de l'insuline (transport du glucose et des acides aminés, altération de l'activation de la glycogène synthétase) concourent à la diminution de la réponse des tissus cibles de l'hormone. Une fois élucidé le mécanisme conduisant à l'hyperinsulinémie on peut penser qu'il sera alors possible de prévenir ou de traiter l'obésité à un stade précoce, lorsque l'insulinorésistance n'est pas encore établie. Les progrès dans la compréhension du mécanisme d'action de l'insuline et dans le rôle éventuel du tissu adipeux brun peuvent également laisser entrevoir la possibilité d'un traitement efficace à un stade plus tardif, chez les obèses insulinorésistants

\section{Summary}

Obesity is often associated with insulin resistance in human and animal obesities. Insulin resistance results from defects at the level of the insulin receptor and from alterations independent from the receptor. 1) Insulin receptor number is decreased in most tissues from obese subjects with, as a consequence, a rightward shift in the insulin doseresponses. 2) Glucose transport, amino acid transport, insulin activation of glycogen synthase are defective in muscles of obese, insulin-resistant animals. These defects could explain the decrease in the biological responses to maximally effective insulin concentrations. 3) Insulin receptor tyrosine kinase activity is altered. The evolutive aspect of the obesity syndrome is also stressed in this review. At the early phase of obesity, there is an hypersecretion of insulin due to hyperphagia and/or central nervous system disorder ; adipose tissue and liver are hyperresponsive to insulin, while muscle is normo or hyporesponsive and obesity rapidly develops. At a later phase, hyperinsulinemia becomes more marked, insulin receptor number is decreased and insulin resistance is established. This corresponds to the static phase of the obesity syndrome. Hyperinsulinemia is clearly responsible for the insulin resistance which occurs in the obesity syndrome. A defect in thermogenesis also probably participates to the development of the obesity syndrome, at least in rodents. A better understanding of the mechanism of insulin action and of the role of the brown adipose tissue should allow an efficient therapy of obesity.

\section{TIRÉS A PART}

Y. Le Marchand-Brustel : Inserm U.145, faculté de médecine, avenue de Valombrose, 06034 Nice Cedex. 